\title{
science \\ Bibliometric analysis of flipped classroom publications from the Web of Science Core Collection published from 2000 to 2019
}

\author{
Hsin-Luen Tsai ${ }^{1,2}$, Jia-Fen $\mathrm{Wu}^{3}$ \\ 'Department of Education, National Kaohsiung Normal University, Kaohsiung, Taiwan; ${ }^{2}$ Bachelor Degree Program in Applied \\ Unmanned Aerial Vehicle Technology, Chang Jung Christian University, Tainan, Taiwan; ${ }^{3}$ Early Childhood Education, School of \\ Education, JingGangShan University, Ji'an, China
}

\section{Abstract}

Purpose: This study analyzed the bibliometric characteristics of flipped classroom publications in the Social Science Citation Index and Science Citation Index Expanded from 2000 to 2019.

Methods: The terms related to "flipped classroom" and "inverted learning" were the keywords for searching journal articles on January 3, 2020.

Results: There are 645 articles (including 33 early-access articles), representing 1,938 authors in the 210 journals scanned. The United States, China, and Taiwan were three leading countries/regions in this field. In the top 10 countries, to 10 institutions, the top eight mostcited journals were identified by either the number of publications or the number of citations. Hot-spot themes from the 24 highly-cited articles and author keyword co-occurrence analysis focus on empirical research in the flipped classroom, the overall feasibility of the flipped classroom course design and practical model, and students' performances, and student-regulated learning (active learning and readiness) outcomes.

Conclusion: TThe results indicate that the United States dominated flipped classroom research, originating most of the highly-cited articles, having more prolific authors, and presenting the most-cited institutions. Furthermore, little research has been undertaken into arriving at an understanding of evidentiary effectiveness or consistency in a flipped classroom. Based on the trends identified, we need a call for more specific types of research into the effectiveness of flipped classroom studies and systematic reviews.

Keywords

Bibliometric analysis; Flipped classroom; Visualization; Keyword co-occurrence, Co-authorship

Correspondence to Jia-Fen Wu wujiafen@jgsu.edu.cn

\section{ORCID}

Hsin-Luen Tsai

https://orcid.org/0000-0002-5532-3451 Jia-Fen Wu

https://orcid.org/0000-0003-1097-221X

\section{Introduction}

Background/rationale: The flipped classroom has recently become an important pedagogical approach for students to learn in an interactive learning environment. Unlike traditional teach- 
ing methods, the concept of flipped classroom refers to the conversion of the roles of teachers and students. Simply put, a flipped classroom approach is a type of blended learning strategy that reverses the traditional learning environment by delivering online learning materials before class, with instructional time to deepen learners' understanding through discussion with peers to facilitate more extensive problem-solving activities. Although the flipped classroom approach has rapidly become the academic focus of education, there is little empirical research on the effectiveness or consistency of flipping classrooms [1].

Perhaps because of the lack of rigorous research design, they still cannot be considered as evidence-based teaching methods even if studies have pointed out that flipped classroom approach can meet the needs of teachers and students [2]. To date, no articles have been published to illustrate the entire picture of flipped classroom-related publications, including the distribution of annual publications, source of authorship, most influential articles.

Purpose: We would like to map flipped classroom knowledge through bibliometric and social network analyses, including annual publications, most-cited countries, most-cited journals, most-cited institutions, most-cited institutions, hot-spot themes, and potential trends.

\section{Methods}

Ethics statement: Neither institutional review board's approval nor informed consent is required because it is a study based on the literature database.

Study design: It is the bibliometric analysis of the specific topic from literature databases.

Data/measurement: Data related to the present study were retrieved from the core collection of Web of Science on January

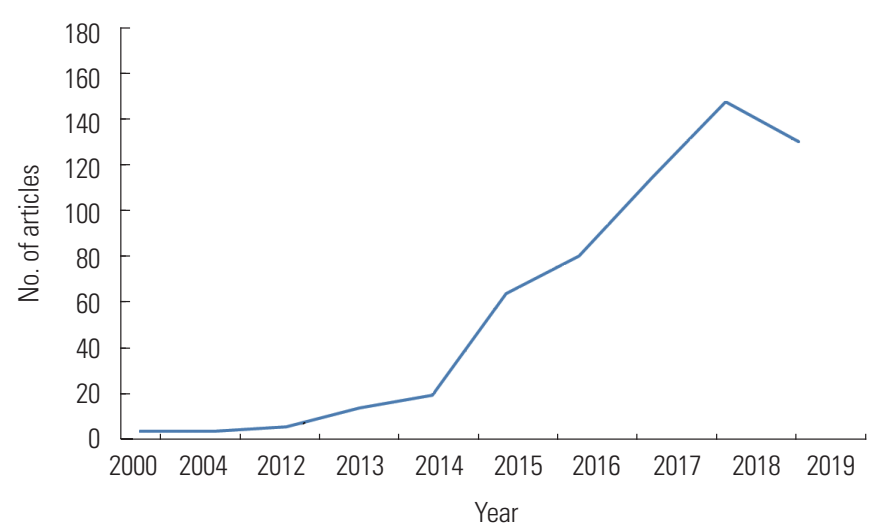

Fig. 1. The distribution of annual publications on the flipped classroom approach.
3, 2020. Twenty-two terms related to "flipped classroom" and "inverted learning" were used as the keywords for searching journal articles. Only those publications published in the years ranging from 1900 to 2019 were considered. The timespan was set as "all year" to thoroughly retrieve related data from the past. There are 645 articles (including 33 early-access articles), representing 1,938 authors in the 210 journals scanned. A total of 15,654 references with 1,440 keywords are included in the titles and abstracts (Dataset 1).

Visualization: Data obtained from the core collection of Web of Science were exported to VOSviewer used to visualize bibliometric maps related to scientific affairs [3] and to estimate the relationships among actors (i.e., author, country, journal, institution) in the given network by centralities and density contour maps.

\section{Results}

As shown in Fig. 1, the first two articles appeared in 2000 and then there was a four-year long pause until 2004. Only two articles were published in 2004 and from then on the growth rate remained null until 2012. Most publications were in 2018. A total of 53 countries were counted and only the top 10 countries were listed. Top 10 countries were presented in Fig. 2. The United States, ranking first, contributed the most original articles (284, $46.5 \%$ of the 611$)$; followed by the China $(78,12.8 \%)$, Taiwan $(74,12.1 \%)$, Spain $(43,7 \%)$, and Australia (40, 6.5\%). The United States had the most citations, followed by Australia, Taiwan, China, and Canada, respectively. Australia ranked second in the number of citations $(1,241)$, followed by Taiwan (992) and China (516). Among the co-authorship relations of the top 10 countries, the three top international co-authorship relations are the United States, Taiwan, and China (Fig. 3).

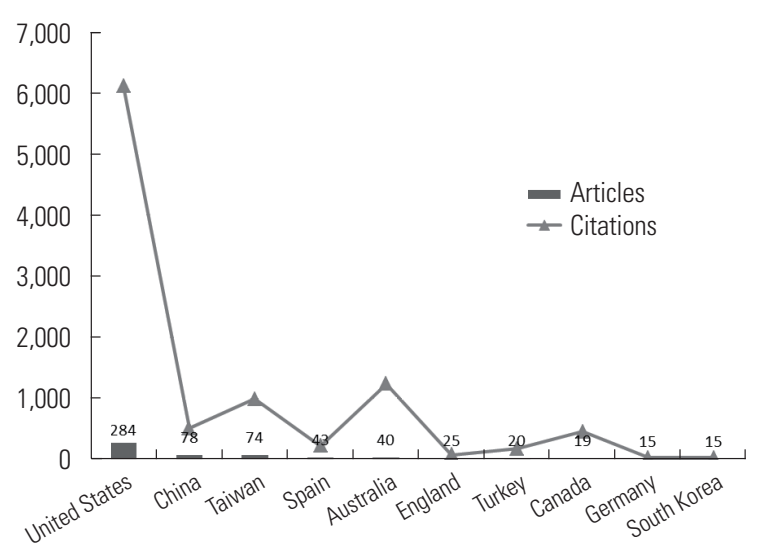

Fig. 2. Top 10 countries [cited on January 3, 2020]. 


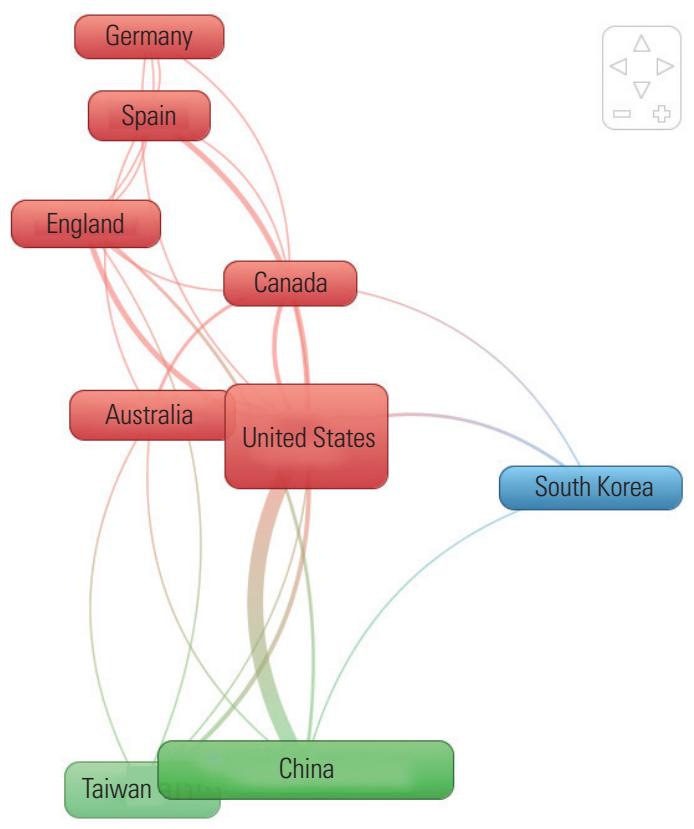

Fig. 3. Bibliometric network of co-authorship relations among top 10 countries.

As shown in Fig. 4, National Taiwan Normal University produced most articles (15 articles), followed by National Taiwan University Science \& Technology (13), University North Carolina (13), University Hong Kong (10), and Harvard Medical School (9). The University of North Carolina ranks first in citations (660), followed by National Taiwan Normal University (182), and National Taiwan University of Science \& Technology (176). The eight most-cited journals with citation of at least 300 in Fig. 5 were Computer \& Education (24), Educational Technology \& Society (22), Internet and Higher Education (5), Journal of Economic Education (6), Higher Education Research \& Development (2), BMC Medical Education (21), American Journal of Pharmaceutical Education (23), IEEE Transactions on Education (6), Academic Medicine (3), and Journal of Chemical Education (25).

In Fig. 6A, lines represent their co-occurrence connection among those keywords shown and the size of each node indicates the number of documents, the larger the node the more documents co-occur. The term flipped classroom is the leading keyword and has stronger links with active learning, blended learning, higher education, and inverted classroom. As shown in the density visualization of Fig. 6B, three hotspot keywords, flipped classroom, active learning and blended learning, are detected to lead the research trend.

As shown in Fig. 7, a visual representation of the top 24 most-cited articles in the past 20 years was extracted by their numerical citations in 210 journals and in the direct citations made between those 645 articles. The most significant articles

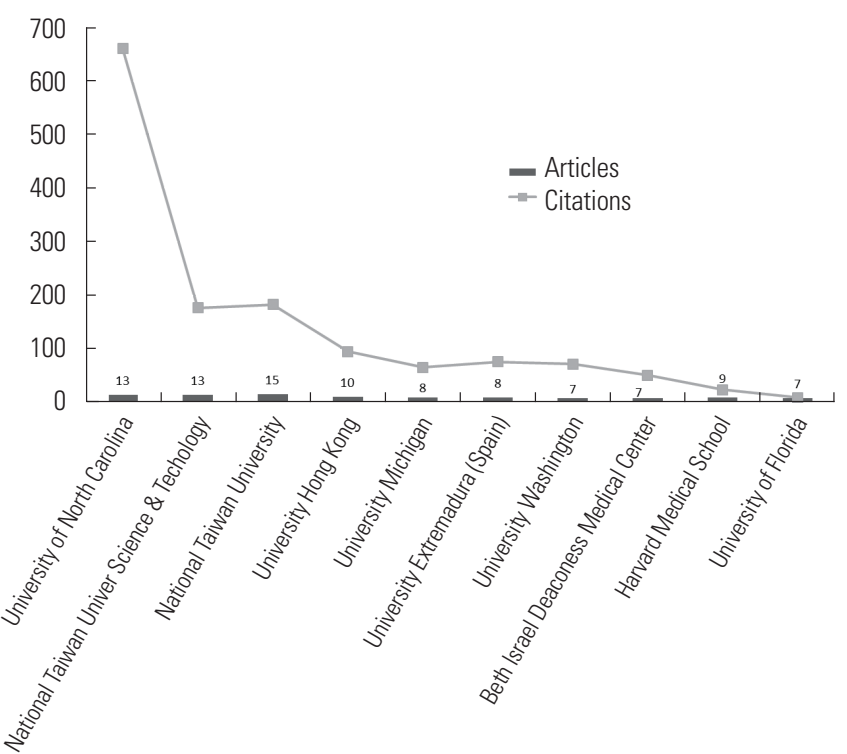

Fig. 4. Top 10 most-cited institutions by articles and citations.

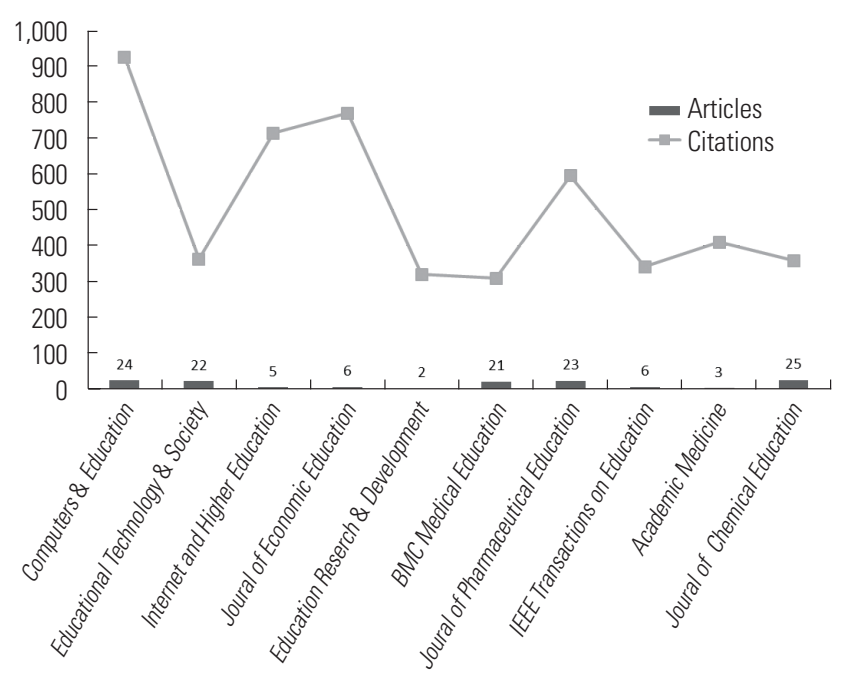

Fig. 5. Top eight most-cited journals with citation of at least 300.

of the 24 most-cited articles were written by Lage and Platt [4], O'flaherty and Phillips [5], McLaughlin et al. [6], Abeysekera and Dawson [1], and Mason et al. [7]. Derived from the 24 most-cited articles, the hot-spot themes on the flipped classroom approach, higher education, redesign course model, students' performance and engagement, and active learning.

\section{Discussion}

A total of 645 flipped classroom-related articles were published during the past 20 years, from 2000 to 2019. The amount of publications gradually increased after 2013 and 


\section{science editing/}
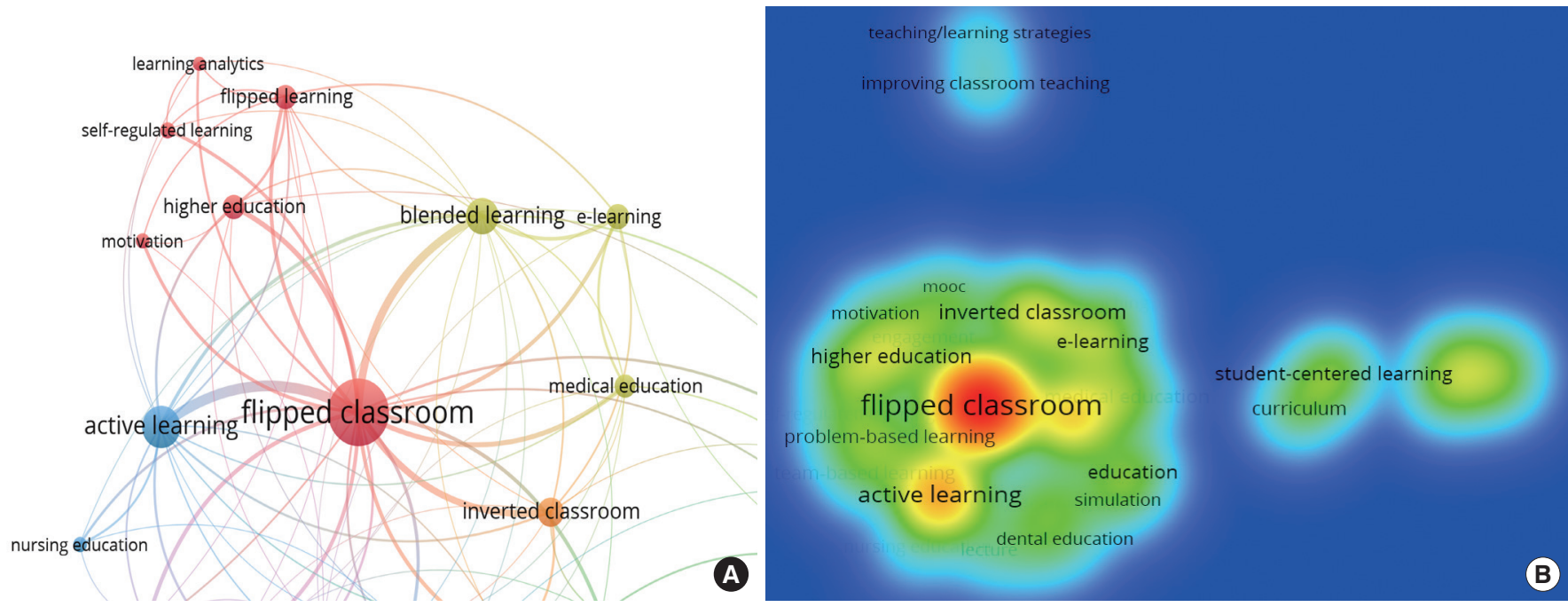

Fig. 6. Visualization map of keyword co-occurrence analysis. (A) Network visualization map based on occurrence-weights and (B) density visualization map based on occurrence-weights.

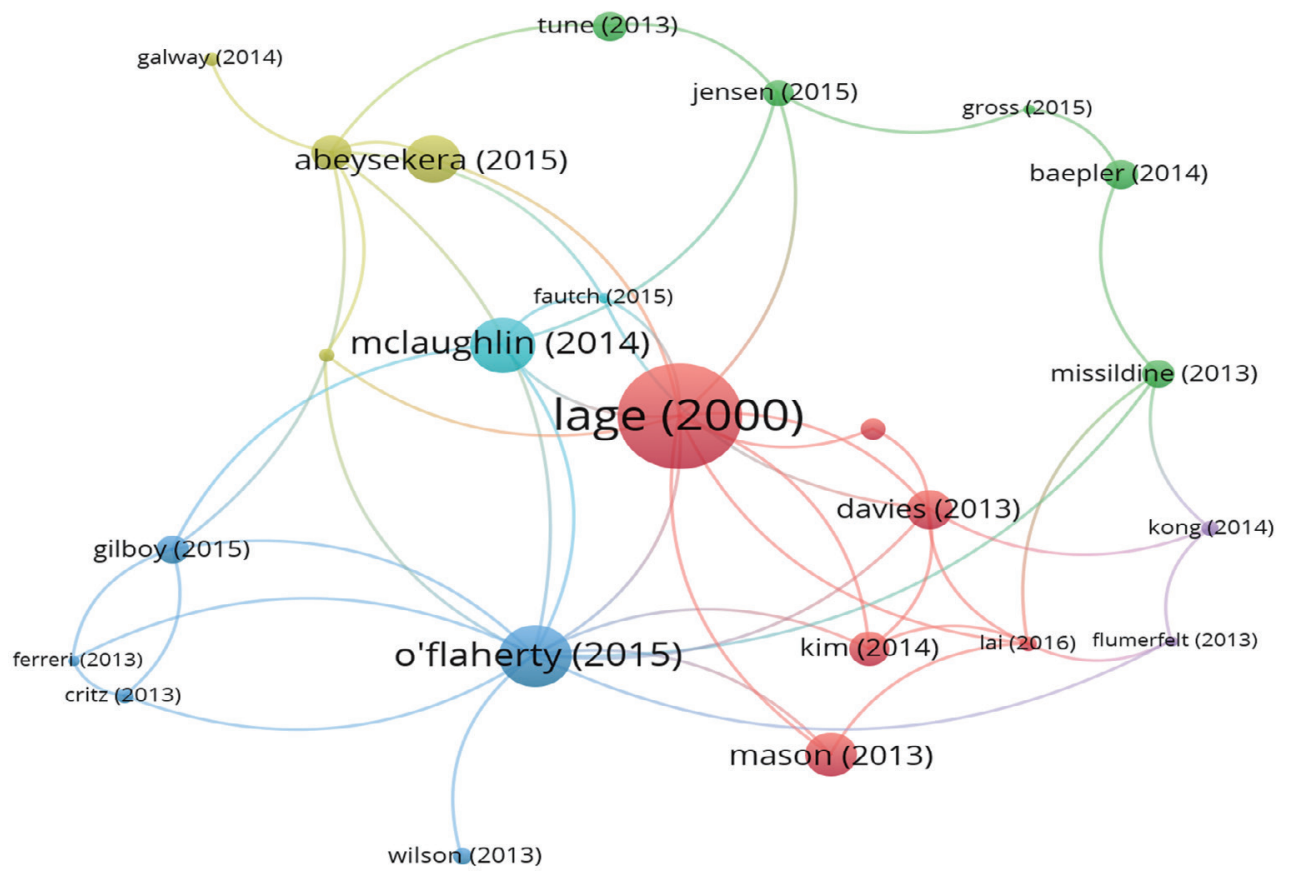

Fig. 7. Bibliometric network map of authors of the 24 most-cited articles.

then dramatically increased from 2015 onwards. The publications between 2013 and 2019 cover $98.7 \%$ of the total of 611 articles (Fig. 1). The most productive publication time was from 2017 to 2018. The United States is the world's leading country with the most publications, most citations, and the highest total link strength, the positive strength of links with other countries (Fig. 2). There was a considerable international collaboration by the United States with eight countries (three Asian countries, except Turkey, three European countries, Canada, and Australia). Taiwan ranked second in total link strength, followed by China, Australia, and Spain. On the other hand, the number of research collaborations with Turkey is zero (Fig. 3). Out of the top ten institutions, three institutions are located in Asia, one in Spain, and six institutions are in the United States (Fig. 4). The University of North Carolina ranks first in citations (660), followed by National Tai- 
wan Normal University (182), and National Taiwan University of Science \& Technology (176). The University of North Carolina also ranks first in Total Link Strength. Indicating its significant impact on co-authorship relations, followed by National Taiwan University of Science \& Technology, National Taiwan Normal University, and the University of Hong Kong.

These top eight most-cited journals in Fig. 5 are attributed to three clusters: Cluster 1 includes education-related journals, such as Computers \& Education, Educational Technology \& Society, Internet and Higher Education and IEEE Transactions on Education; Cluster 2 includes medically-related journals, such as BMC Medical Education, American Journal of Pharmaceutical Education, and Academic Medicine; Cluster 3 includes higher education-related journals, such as Higher Education Research \& Development and Journal of Chemical Education.

Authors' keywords with higher centralities are closer to the core concept of the flipped classroom approach. A keyword co-occurrence analysis network is investigated to understand the co-occurrence of keywords in global flipped classroom publications at the micro level. Authors' keywords reflect the core content of a publication [8], and they make a paper searchable and then lead to a successful search reference resource [9]. A keyword co-occurrence analysis network is investigated to understand the co-occurrence of keywords in global flipped classroom publications at the micro-level. Those author keywords in Fig. 6 indicate that flipped classroom, also known as an inverted classroom, is an approach to improve active learning in higher education using blended learning strategy.

The leading article "Inverting the classroom: a gateway to creating an inclusive learning environment" [10] explained the evidence of a mismatch between an instructor's teaching style and a student's learning style to illustrate the scenario of the inverted classrooms for readers. Most-cited articles in 2013 focus on either case studies in various subjects (i.e., chemistry, statistics, nursing education, physiology) for at-risk students and college students or comparison studies to prove the positive effects of flipped classrooms on students' performance and engagement. The articles in 2014 present the second theme that flipped classroom model or course redesign is useful to foster students' active learning, performance, and engagement. Articles in 2015 center on students' motivation and perceptions towards implementing the flipped classroom approach for most researchers who believe that the positive effects found in this approach will contribute significantly to students' outstanding performance and great satisfaction.

In 2016, the research focus has been shifted forward from students' surface performance to self-regulated learning readiness and retention. For example, Ryan and Reid [11] men- tioned students' performance and retention in the surgery core clerkship flipped classroom; McLean et al. [12] state that students learning in flipped classrooms do not just obtain surface gains; Hao [13] brings up "learning readiness" which is pivotal for the success of implementing the flipped classroom approach. Lai and Hwang's study [14] provides a self-regulated flipped classroom and presents the transformative potential and different research focus from the past. The tailored flipped classroom model is emphasized to meet the requirement and needs for different subjects to be instructed [11,14].

Hot-spot themes from the highly-cited articles and author keyword co-occurrence analysis focus on empirical research in the flipped classroom, the overall feasibility of the flipped classroom course design and practical model, and students' performances, and student-regulated learning (active learning and readiness) outcomes.

The results of this study indicate that the United States dominated flipped classroom research, originating most of the highly-cited articles, having more prolific authors, and presenting the most-cited institutions. Furthermore, emerging hot-spot themes from the highly-cited articles and author keyword co-occurrence analysis focus on empirical research in the flipped classroom, the overall feasibility of the flipped classroom course design and practical model, and students' performances, and student-regulated learning (active learning and readiness) outcomes, which are likely to be paid more attention in the future. Despite flipped classroom approaches having recently been the subject of much popular attention in the media, Google Search, etc., little research has been undertaken into arriving at an understanding of evidentiary effectiveness or consistency in a flipped classroom. After all, flipped classroom approach is not a panacea, nor is it a one-size-fit-all method. The studies suggest a call for more specific types of research into the effectiveness of flipped classroom studies and systematic reviews which may illustrate the entire picture of the flipped classroom approach as a whole. These insights into the exhibited priorities and trends of recent (past two decades) research may assist future academic pursuits leading to further adoption of the flipped learning approach.

\section{Conflict of Interest}

No potential conflict of interest relevant to this article was reported.

\section{Acknowledgments}

This work was supported by the funding agency in Jiangxi Province Higher Education Teaching Reform Research in 2019 (JXJG-19-9-17). 


\section{Data Availability}

The dataset file is available from the Harvard Dataverse at: https://doi.org/10.7910/DVN/39TUY4

Dataset 1. Add raw data of the 645 articles with references and keywords

\section{References}

1. Abeysekera L Dawson P. Motivation and cognitive load in the flipped classroom: definition, rationale, and a call for research. High Educ Res Dev 2015;34:1-14. https://doi.org /10.1080/07294360.2014.934336

2. Triana P. Evidence-based policy: a realist perspective. London: Sage Publications; 2006. https://doi.org/10.1080/ 15588740802262039

3. van Eck NJ, Waltman L. Software survey: VOSviewer, a computer program for bibliometric mapping. Scientometrics 2010;84:523-8. https://doi.org/10.1007/s11192-009-0146-3

4. Lage MJ, Platt G. The internet and the inverted classroom. J Econ Educ 2000;31:11. https://doi.org/10.2307/1183335

5. O'flaherty J, Phillips C. The use of flipped classrooms in higher education: a scoping review. Internet High Educ 2015;25:85-95. https://doi.org/10.1016/j.iheduc.2015.02.002

6. McLaughlin JE, Roth MT, Glatt DM, et al. The flipped classroom: a course redesign to foster learning and engagement in a health professions school. Acad Med 2014;89:236-43. https://doi.org/10.1097/ACM.0000000000000086

7. Mason GS, Shuman TR, Cook KE. Comparing the effec- tiveness of an inverted classroom to a traditional classroom in an upper-division engineering course. IEEE Trans Educ 2013;56:430-5. https://doi.org/10.1109/TE.2013.2249066

8. Xing C, Wang Y, Liu H. A scientometrics review on nonpoint source pollution research. Ecol Eng 2017;99:400-8. https://doi.org/10.1016/j.ecoleng.2016.11.028

9.Babaii E, Taase Y. Author-assigned keywords in research articles: Where do they come from? Iranian Journal of Appl Ling 2013;16:1-19.

10. Lage MJ, Platt GJ, Treglia M. Inverting the classroom: a gateway to creating an inclusive learning environment. J Econ Educ 2000;31:30-43. https://doi.org/10.2307/1183338

11. Ryan MD, Reid SA. Impact of the flipped classroom on student performance and retention: a parallel controlled study in general chemistry. J Chem Educ 2016;93:13-23. https://doi.org/10.1021/acs.jchemed.5b00717

12. McLean S, Attardi SM, Faden L, Goldszmidt M. Flipped classrooms and student learning: not just surface gains. Adv Physiol Educ 2016;40:47-55. https://doi.org/10.1152/ advan.00098.2015

13. Hao Y. Exploring undergraduates' perspectives and flipped learning readiness in their flipped classrooms. Comput Hum Behav 2006;59:82-92. https://doi.org/10.1016/j.chb. 2016.01.032

14. Lai CL, Hwang GJ. A self-regulated flipped classroom approach to improving students' learning performance in a mathematics course. Comput Educ 2016;100:126-40. https:// doi.org/10.1016/j.compedu.2016.05.006 